\title{
TEILNEHMER DER TAGUNG
}

Prof. Dr. Horst Baier, Renkenweg 9, D-7750 Konstanz

Prof. Dr. Ernst Behler, Comparative Literature GN-32, University of Washington, Seattle, Washington 98195/USA

Prof.Dr. Rudolph Berlinger, Residenzplatz 2, D-8700 Würzburg

Prof. D Dr. Eugen Biser, Kaulbachstr. 31/I, D-8000 München 22

Prof. Dr. Mihailo DJurić, Banijska 1, YU-11000 Beograd

Prof. Dr. Sander L. Gilman, Department of German Literature, Cornell University, Goldwin Smith Hall, Ithaca, N.Y. 14853/USA

Prof. Dr. Georges Goedert, 40, rue Schrobilgen, Luxembourg, Großherzogtum Luxemburg

Prof. Dr. Karlfried Gründer, Schattbachstr. 4, D-4630 Bochum-Querenburg

Prof. Dr. Peter Heller, State University of New York at Buffalo, Germanic-Slavic, Wilkeson-Quad, SUNY/Buffalo, N.Y., 14214/USA

Curt Paul Janz, Brühlweg 42, CH-4132 Muttenz

Prof. Dr. Walter Kaufmann, Princeton University, Department of Philosophy, 1879 Main Hall, Princeton, New Jersey 08540/USA

Prof. Dr. Frederick R. Love, Brown University, Department of German, Box E, Providence, Rhode Island 02912/USA

Prof. Dr. Reinhart Maurer, Ithweg 1, D-1000 Berlin 37

Prof. Dr. C. A. Milier, Yacht Haven, Friday Harbor, San Juan Island, Washington 98250/USA

Prof. Dr. Mazzino Montinari, via Gabriele d'Annunzio, 237, I-50132 Florenz

Prof. Dr. Wolfgang Müller-Lauter, Adolf-Martens-Str. 11, D-1000 Berlin 45

Prof. Dr. Karl PestalozzI, Universität Basel, Strengigässli 17, CH-4123 Allschwil

Dr. Jörg Salaquarda, Bozener Str. 10, D-1000 Berlin 62

Prof. Dr. Hans-Martin SAss, Klein-Herbeder-Str. 9a, D-5810 Witten-Heven

Prof. Dr. Richard L. SchACHT, University of Illinois at Urbana-Champaign, Department of Philosophy, Urbana Illinois 61801/USA

Prof. Dr. David S. Thatcher, University of Victoria, Department of English, Post Office Box 1700, Victoria, B. C., Canada V8W2Y2

Prof. Dr. Carl Ulmer, Universitätsstr. 7/II/II, A-1010 Wien

Prof. Dr. Paul Valadier, 128, rue Blomet, F-75015 Paris

Prof. Dr. Heinz Wenzel, Harnackstr. 16, D-1000 Berlin 33 
